\title{
Malignant Intracranial Meningioma With Spinal Metastasis
}

\author{
-Case Report-
}

\author{
Hiroki Kuroda, Hiroshi KASHIMURA, Kuniaki OgASAWARA, \\ Atsushi SugaWARA, Masayuki SASOH, Hiroshi ARAI, and Akira OGAWA
}

Department of Neurosurgery, Iwate Medical University School of Medicine, Morioka, Iwate

\begin{abstract}
A 48-year-old woman presented with a left cerebellopontine angle mass. Over a 93-month period, the patient underwent seven surgeries, two radiosurgeries, and one external beam radiotherapy. The tumor was histologically benign at the first operation, but exhibited unusually aggressive behavior after failed radiosurgery and demonstrated clinical characteristics of malignancy such as spinal metastasis. The patient underwent gamma knife radiosurgery (GKR) for recurrence after the first operation, despite the tumor being located in a resectable region. The tumor did not respond. Six months after the sixth surgery, the patient presented with progressive lower extremity paraparesis and sensory disturbance below the T11 dermatome. Magnetic resonance imaging revealed multiple intradural mass lesions located at the T2, T11-T12, and L2 levels. She died 4 months after the diagnosis of spinal metastases. Retrospectively, we speculate that if a tumor is located in a resectable region and Simpson grade I or II tumor resection is possible, direct surgery may be a safer option than GKR.
\end{abstract}

Key words: cerebrospinal fluid, meningioma, spinal metastasis

\section{Introduction}

Metastasis from meningioma is rare, accounting for only about $0.1 \%$ of all meningiomas. ${ }^{18)}$ The most common sites of metastasis are the lungs, liver, lymph nodes, and bones. ${ }^{10)}$ Seeding of meningioma cells through the cerebrospinal fluid (CSF) pathways with subsequent establishment of metastatic implants is rare. We describe a case of metastasis of meningioma through the CSF pathway after seven surgical and two radiosurgical interventions.

\section{Case Report}

A 48-year-old woman suffered progressive headache and was referred to our institution. Magnetic resonance (MR) imaging revealed a mass lesion located in the left cerebellopontine angle, with heterogeneous enhancement with gadolinium (Fig. 1).

First operation: The patient underwent a left lateral suboccipital craniotomy and the tumor was removed with Simpson grade II resection (complete macroscopic removal). Histological examination

Received April 8, 2008; Accepted January 15, 2009
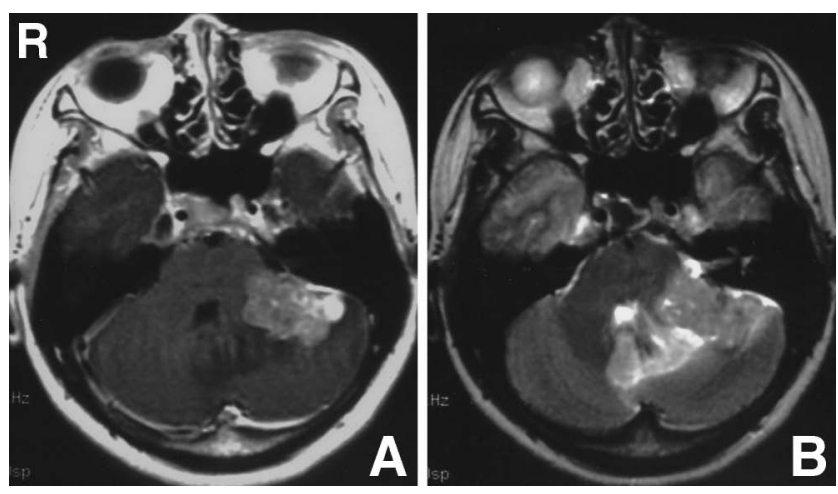

Fig. 1 Axial $\mathrm{T}_{1}$-weighted with contrast medium (A) and $T_{2}$-weighted (B) magnetic resonance images showing a tumor located in the left cerebellopontine angle, with lobulated margins (A) and perifocal edema (B).

revealed meningothelial meningioma, and MIB-1 staining index was $5.5 \%$ (Fig. $2 \mathrm{~A}$ ). The postoperative course was uneventful, and the patient was discharged without neurological deficits. MR imaging performed 36 months later revealed tumor recurrence in the region corresponding with the primary 


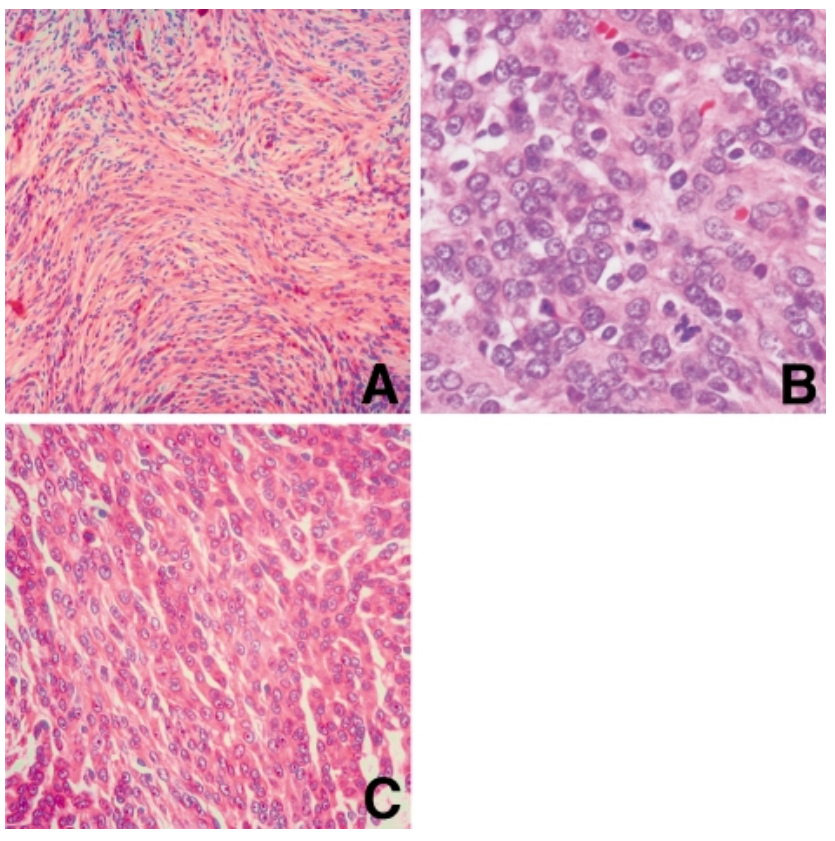

Fig. 2 A: Photomicrograph of the specimen obtained at the first operation showing the entire field consists of tumor cells, with MIB-1 staining index of $5.5 \%$. Hematoxylin and eosin stain, original magnification $\times 200$. B: Photomicrograph of the specimen obtained at the third operation showing obvious atypical features including patternless growth, a high nuclear/cytoplasmic ratio, and frequent mitoses (16/10 high power fields), with MIB-1 staining index of 36.7\%. Hematoxylin and eosin stain, original magnification $\times 400$. C: Photomicrograph of the specimen obtained at the seventh operation showing cellular atypia and mitotic figures, with MIB-1 index of $53.1 \%$. Hematoxylin and eosin stain, original magnification $\times \mathbf{2 0 0}$.

\section{lesion.}

First gamma knife radiosurgery (GKR): GKR was performed for the recurrent tumor, 42 months after the first operation. The marginal dose corresponding to the $50 \%$ isodose line was $14 \mathrm{~Gy}$, and the maximal dose was $28 \mathrm{~Gy}$. MR imaging performed 23 months later revealed further tumor growth (Fig. 3A).

Second operation: The tumor was removed with Simpson grade II resection (complete macroscopic removal of the tumor and its visible extensions), 22 months after the first GKR. Histological examination confirmed meningothelial meningioma, and MIB-1 staining index had increased to $32.3 \%$. MR imaging performed 11 months after the second operation revealed a recurrent tumor located more ven-

Neurol Med Chir (Tokyo) 49, June, 2009

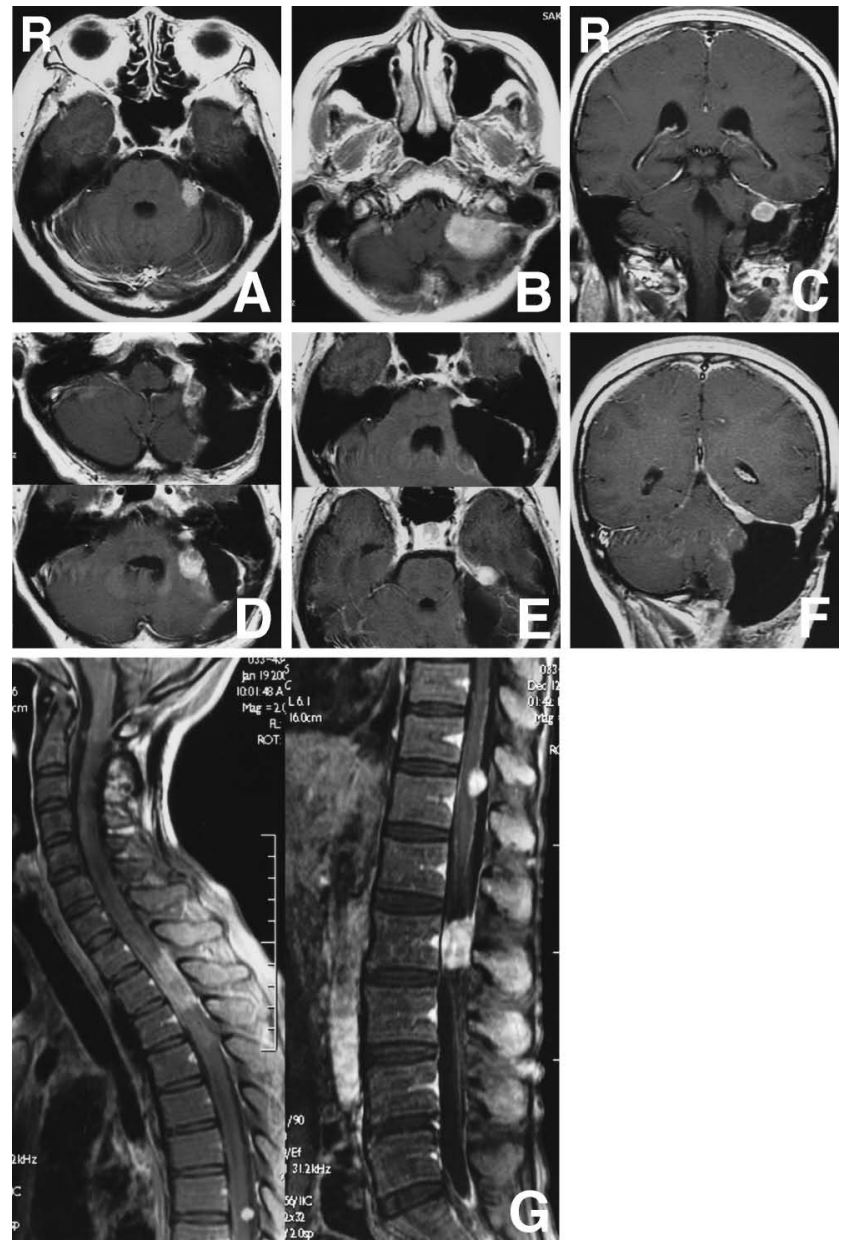

Fig. 3 Magnetic resonance (MR) images after the first (A), the second (B), the third (C), the fourth (D), the fifth (E), the sixth operation $(F)$ and the second gamma knife radiosurgery (G), respectively. Axial $\mathrm{T}_{1}$-weighted MR image with contrast medium showing tumor recurrence in the region corresponding with the primary lesion (A). Axial $T_{1}$-weighted MR image with contrast medium showing a recurrent tumor located more ventrally and caudally than the initial lesion (B). Coronal $T_{1}$-weighted $M R$ image with contrast medium showing a recurrent tumor at the cerebellar surface and below the cerebellar tent (C). Axial $T_{1}$-weighted MR images with contrast medium showing recurrent tumor at the cerebellar surface and within the internal auditory canal and jugular foramen (D). Axial $\mathrm{T}_{1}$-weighted MR images with contrast medium showing a recurrent tumor in the internal auditory canal and extending to the left temporal base (E). Coronal $T_{1}$-weighted $M R$ image with contrast medium showing regrowth of the residual tumor on the cerebellar tent (F). Spinal sagittal $T_{1}$-weighted MR images with contrast medium showing multiple intradural enhanced mass lesions at the T11T12 and L2 levels (G). 
trally and caudally than the initial lesion (Fig. 3B).

Third operation: Ten months after the second operation, the tumor was again removed with Simpson grade II resection because of the dural attachment to the sigmoid sinus wall. Histological examination revealed atypical meningioma, with MIB-1 staining index of $36.7 \%$ (Fig. 2B). MR imaging performed 2 weeks later revealed a recurrent tumor at the cerebellar surface and below the cerebellar tent (Fig. 3C).

Fourth operation: The tumor was removed, with parts of the cerebellar tent and the dura mater, with Simpson grade II resection, 2 months after the third operation. Part of the cerebellar tent and its dural attachments were removed as widely as possible. Histological examination revealed anaplastic meningioma, with nuclear polymorphism and numerous mitoses. MIB-1 staining index was $40 \%$. MR imaging performed 10 days later revealed further tumor recurrence at the cerebellar surface and within the internal auditory canal and jugular foramen (Fig. 3D).

Fifth operation: Further tumor removal with Simpson grade II resection was performed, 2 months after the fourth operation. The tumor had extended to involve cranial nerves VII and VIII and the lower cranial nerves. The lower cranial nerves could be preserved, but the cranial nerves VII and VIII were resected with the tumor. Histological examination confirmed anaplastic meningioma with MIB-1 staining index of $32 \%$. MR imaging performed 2 months later revealed a recurrent tumor in the internal auditory canal with extension to the left temporal base (Fig. 3E).

Sixth operation: The tumor was removed with Simpson grade III resection, 4 months after the fifth operation. The tumor extended to the lower cranial nerves and left vertebral artery. The lower cranial nerves were resected with the tumor, but the tumor in the surrounding vertebral artery could not be resected. Histological examination confirmed anaplastic meningioma, with MIB-1 staining index of $38 \%$. MR imaging performed 7 days later revealed regrowth of the residual tumor (Fig. 3F).

Second GKR: GKR and additional external beam radiotherapy $(60 \mathrm{~Gy} / 30$ fractions, one fraction per day for 7 weeks) were performed immediately after the sixth operation. Six months after the second GKR, the patient presented with progressive lower extremity paraparesis and sensory disturbance below the T11 dermatome. MR imaging revealed multiple intradural mass lesions at the T2, T11-T12, and L2 levels (Fig. 3G).

Seventh operation: Laminectomy was performed from T11 to L2 and the intradural extramedullary tumors at the T11-T12 and L2 levels were removed, 7 months after the sixth operation. The tumors were located on the surface of the spinal cord dura mater but without attachment. Histological examination confirmed anaplastic meningioma, with MIB-1 staining index of $53.1 \%$ (Fig. 2C). MR imaging performed one month later showed enlargement of the T2 tumor mass and development of a tumor at the T7 level. Finally, the patient's consciousness deteriorated progressively and she died 93 months after the first operation.

\section{Discussion}

Only 18 cases of metastasis of intracranial meningioma to the spine have been reported.1,3,4,6-9,11-14,16,19) The risk of CSF dissemination of this tumor is not clear. Twelve of these 18 patients had undergone two or more surgical procedures. In contrast, 4 of 12 patients with metastatic meningiomas disseminated through the CSF had not undergone craniotomy. ${ }^{10)}$ Therefore, surgical mobilization of the tumor cells does not seem to be important in the pathogenesis of CSF metastasis. Four of 200 consecutive patients with meningioma exhibited spinal metastasis. ${ }^{3)}$ All four patients had received extensive prior therapy, including surgery (often multiple), radiotherapy (often both external beam radiotherapy and stereotactic), and chemotherapy. Survival was 6 months and 11 months in two of the four patients, while the other two patients are alive at the last follow up. The survival of patients after diagnosis of spinal metastases was reported as 6 and 8 months. ${ }^{1,9)}$

In the present case, the meningioma was histologically classified as benign at the first operation, but exhibited unusually aggressive behavior after failed radiosurgery, and later exhibited clinical characteristics of malignancy such as spinal metastasis. Radiosurgery is an effective treatment modality for patients with intracranial meningiomas, as proven by excellent short-term radiosurgically demonstrated control rates, which approach or exceed $90 \%$. However, little is known about the GKR-resistant meningioma that sometimes occurs, and skull base meningioma growth can be aggressive after failed radiosurgery in some patients. ${ }^{5}$ Our patient underwent GKR for the recurrent tumor after the first operation, despite the tumor being located in a resectable region. The tumor did not respond to GKR. The histology remained unchanged for almost 2 years after GKR, but the MIB-1 staining index definitely increased compared to that at the first operation. MIB-1 staining index is useful for evaluating cell kinetics, ${ }^{2,7,17)}$ so such an increase suggests that the tumor should have been considered biologi- 
cally aggressive after GKR. However, the MIB-1 staining index obtained at the first operation was definitely higher than usual. Meningiomas with a MIB-1 staining index of $3 \%$ or more have a significantly higher tendency for recurrence than those with lower MIB-1 staining index. ${ }^{15)}$ Thus, progression of the tumor may have resulted from the natural course of the disease, rather than representing an adverse effect of GKR. Retrospectively, we speculate that if a tumor is located in a resectable region and Simpson grade I or II tumor resection is possible, direct surgery is a safer option than GKR.

\section{References}

1) Akimura T, Orita T, Hayashida O, Nishizaki T, Fudaba $\mathrm{H}$ : Malignant meningioma metastasizing through the cerebrospinal pathway. Acta Neurol Scand 85: 368-371, 1992

2) Arai H, Beppu T, Wada T, Yoshida Y, Kubo Y, Suzuki M, Ogawa A: Pathological analyses of early recurrence and malignant transformation in meningiomas. Brain Tumor Pathol 15: 37-40, 1998

3) Chamberlain MC, Glantz MJ: Cerebrospinal fluid-disseminated meningioma. Cancer 103: 1427-1430, 2005

4) Chuang HC, Lee HC, Cho DY: Intracranial malignant meningioma with multiple spinal metastases. A case report and literature review: case report. Spine 31: E1006-1010, 2006

5) Couldwell WT, Cole CD, Al-Mefty O: Patterns of skull base meningioma progression after failed radiosurgery. J Neurosurg 106: 30-35, 2007

6) Hoffmann GT, Earle KM: Meningioma with malignant transformation and implantation in the subarachnoid space. J Neurosurg 17: 486-492, 1960

7) Iwaki T, Takeshita I, Fukui M, Kitamura K: Cell kinetics of the malignant evolution of meningothelial meningioma. Acta Neuropathol (Berl) 74: 243-247, 1987

8) Kalm H: Ein malignes Tentoriummeningeom mit Metastasierung in die Medulla oblongata und in die subarachnoidalen Liquorraume. Dtsch Z Nervenheilkd 163: 131-140, 1950 (Ger)

9) Kamiya K, Inagawa T, Nagasako R: Malignant in- traventricular meningioma with spinal metastasis through the cerebrospinal fluid. Surg Neurol 32: 213-218, 1989

10) Kepes JJ: Meningiomas. Biology, Pathology, and Differential Diagnosis. New York, Masson, 1982, pp 190-200

11) Kleinschmidt-DeMasters BK, Avakian JJ: Wallenberg syndrome caused by CSF metastasis from malignant meningioma. Clin Neuropathol 4: 214-219, 1985

12) Lee TT, Landy HJ: Spinal metastases of malignant intracranial meningioma. Surg Neurol 50: 437-441, 1998

13) Ludwin SK, Conley FK: Malignant meningioma metastasizing through the cerebrospinal pathways. J Neurol Neurosurg Psychiatry 38: 136-142, 1975

14) Ludwin SK, Rubinstein LJ, Russell DS: Papillary meningioma: A malignant variant of meningioma. Cancer 36: 1363-1373, 1975

15) Matsuno A, Fujimaki T, Sasaki T, Nagashima T, Ide $\mathrm{T}$, Asai A, Matsuura R, Utsunomiya $\mathrm{H}$, Kirino $\mathrm{T}$ : Clinical and histopathological analysis of proliferative potentials of recurrent and non-recurrent meningiomas. Acta Neuropathol 91: 504-510, 1996

16) Miller AA, Ramsden F: Malignant meningioma with extracranial metastases and seeding of the subarachnoid space and the ventricles. Pathol Eur 7: 167-175, 1972

17) Ohta M, Iwaki T, Kitamoto T, Takeshita I, Tateishi J, Fukui M: MIB1 staining index and scoring of histologic features in meningioma. Cancer 74: 3176-3189, 1994

18) Russel DS, Lubinstein LJ: Pathology of the Tumors of the Nervous System. London, Edward Arnold, 1989, pp 430-437

19) Shuangshoti S, Hongsaprabhas C, Netsky MG: Metastasizing meningioma. Cancer 26: 832-841, 1970

Address reprint requests to: Hiroshi Kashimura, M.D., Department of Neurosurgery, Iwate Medical University School of Medicine, 19-1 Uchimaru, Morioka, Iwate 020-8505, Japan.

e-mail: hkashi@iwate-med.ac.jp 